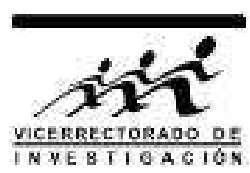

\title{
Estudio numérico del sismo lento y maremoto de Chimbote 1996 (7.6 Mw)
}

\author{
C. Jiménez ${ }^{* 1,2}$, M. Calvo ${ }^{1}$ y H. Tavera ${ }^{3}$ \\ ${ }^{1}$ Facultad de Ciencias Físicas, Universidad Nacional Mayor de San Marcos, Lima, Perú \\ ${ }^{2}$ División de Geofísica, Dirección de Hidrografía y Navegación, Lima, Perú \\ ${ }^{3}$ Area de Sismología, Instituto Geofísico del Perú, Lima, Perú
}

Recibido 09 abril 2015 - Aceptado 23 julio 2015

\begin{abstract}
El 21 de febrero de 1996 un sismo sacudió la región norte del Perú. Este evento generó un maremoto con olas de 2 a $3 \mathrm{~m}$ de altura. Del análisis y el procesamiento de las señales de banda ancha de 15 estaciones telesísmicas, se realizó la inversión de las formas de onda para obtener el mecanismo focal y la función temporal de la fuente, que indica un proceso de ruptura complejo. La duración total del proceso de ruptura fue de $75 \mathrm{~s}$, un valor bastante alto para un sismo relativamente pequeño. El momento sísmico escalar calculado fue de $3.8 \times 10^{20} \mathrm{Nm}$, equivalente a una magnitud de momento de 7.6 Mw. La distribución de la dislocación es heterogénea y consistente con la función temporal de la fuente, con una dislocación máxima de $6.6 \mathrm{~m}$ en torno a la aspereza principal concentrada en un área de $30 \times 30 \mathrm{~km}^{2}$. Se calculó el campo de deformación cosísmico a partir de 28 subfuentes, el cual se utilizó como condición inicial de la propagación del maremoto. Se calcularon los mareogramas sintéticos para Chimbote y Salaverry con alturas máximas de $1.8 \mathrm{~m}$ y $1.0 \mathrm{~m}$, respectivamente.

Palabras claves: Sismo lento, modelo numérico, maremoto.
\end{abstract}

\section{Numerical study of the 1996 Chimbote slow earthquake and tsuna- $\mathrm{mi}(7.6 \mathrm{Mw})$}

On February 21, 1996, an earthquake shook the northern region of Peru. This event generated a tsunami with 2-3 m height waves. From the analysis and the signal processing of 15 broadband teleseismic stations, a waveform inversion was performed to obtain the focal mechanism and source time function indicating a complex rupture process. The total duration of the rupture was $75 \mathrm{~s}$, a rather high value for a relatively small earthquake. The calculated scalar seismic moment was $3.8 \times 10^{20} \mathrm{Nm}$, which corresponds to a moment magnitude of $7.6 \mathrm{Mw}$. The slip distribution is heterogeneous, consistent with the source time function, with a maximum slip of $6.6 \mathrm{~m}$ around the main asperity concentrated in an area of $30 \times 30 \mathrm{~km}^{2}$. Coseismic deformation field was calculated for 28 sub-faults, which was used as the initial condition of the tsunami propagation. Synthetics mareograms were calculated for Chimbote and Salaverry with maximum heights of $1.8 \mathrm{~m}$ and 1.0 $\mathrm{m}$, respectively.

Keywords: Slow earthquake, numerical modeling, tsunami.

${ }^{*}$ cjimenezt@unmsm.edu.pe 
El sismo de Chimbote, Ancash, Perú, de 1996 fue un evento atípico en el sentido que a pesar de su magnitud, 7.6 Mw, relativamente pequeña para provocar un maremoto y con una intensidad máxima de IV en la escala de Mercalli Modificada [1], generó un maremoto con efectos mayores a lo que se esperaba. Como efecto del impacto del maremoto con olas entre 2 y $3 \mathrm{~m}$ de altura, murieron 12 personas principalmente en la desembocadura del río Santa, al norte de Chimbote [1]. Este tipo de evento es conocido en la literatura como sismo tsunamigénico o tsunami earthquake [2], siendo estudiado y clasificado como tal por Okal y Newman [3].

El cálculo del mecanismo focal de la fuente sísmica utlizando 54 lecturas de polaridad de la onda $\mathrm{P}$, para luego realizar la inversión de las ondas telesísmicas de banda ancha para una fuente puntual, caracterizando el proceso de ruptura como múltiple o complejo, fue establecido por Tavera [4]. Asimismo, Ihmle y colaboradores [5], realizaron la inversión de señales de banda ancha filtradas entre los $2 \mathrm{~s}$ y $100 \mathrm{~s}$, para calcular la distribución de la fuente sísmica para un modelo de estructura de velocidades homogéneo. El filtro pasabanda aplicado, elimina la información relevante de componentes de frecuencias mayores a $0.01 \mathrm{~Hz}$, característico de los sismos de ruptura lenta o tsunami earthquake, como es el caso del sismo de Chimbote de 1996, lo que produciría valores subestimados del momento sísmico escalar y de la duración del proceso de ruptura.

Heinrich y colaboradores [6], realizaron el modelado numérico del maremoto generado, en base a la fuente sísmica calculada por Ihmle y colaboradores [5]. Por otro lado, Bourgeois y colaboradores [7], realizaron observaciones de campo para obtener datos del maremoto tales como la máxima inundación horizontal y vertical, runup; también realizaron el modelado numérico del maremoto usando una fuente sísmica homogénea de dimensiones $120 \mathrm{~km} \times 60 \mathrm{~km}$ para una batimetría con resolución de 5 min en altamar y $600 \mathrm{~m}$ cerca a la costa. El modelo lineal utilizado muestra una correlación con los datos de runup observados, debido a que las condiciones de contorno del modelo lineal sobre-estiman los valores de runup observado como un proceso no-lineal, de modo que una fuente simulada con una pequeña dislocación o slip puede provocar un maremoto simulado con un gran runup.

Adicionalmente, Ocola y Huaco (2006) [1] realizaron observaciones de campo desde Huacho, en Lima, hasta la playa Huanchaquito, en La Libertad, reportando datos del maremoto como: máxima inundación vertical, runup, y horizontal, así como los efectos del impacto del maremoto en puertos y localidades costeras. Sin embargo, no realizaron cálculos de modelado numérico.

En el presente trabajo, se realizó la inversión de las formas de onda telesísmicas de banda ancha filtradas en el rango de los $10 \mathrm{~s}$ y $500 \mathrm{~s}$, utilizando un modelo de estructura de velocidades de la onda sísmica de $6 \mathrm{ca}-$ pas más otra capa adicional correspondiente al cuerpo de agua del Océano Pacífico, a fin de modelar el comportamiento de las ondas sísmicas en el oceáno en la vecindad de la fuente. Se calculó la deformación cosísmica inicial en base a la teoría de Okada 8. Además se modeló la propagación del maremoto con una fuente sísmica heterogénea para una batimetría con resolución de $0.5 \mathrm{~min}$ en altamar y a $30 \mathrm{~m}$ cerca a la costa, obteniéndose mareogramas sintéticos en la bahía de Chimbote y en el puerto de Salaverry.

\section{Procedimientos}

El desarrollo de un modelo de velocidades de ondas sísmicas en la vecindad de la fuente, así como la disponibilidad de datos experimentales de batimetría y de un modelo numérico no-lineal de simulación de maremotos, que incluyen periodos más largos de los registros, permiten realizar el modelo numérico del sismo y del maremoto de Chimbote 1996 con mayor detalle.

\begin{tabular}{lll}
\hline Institución & IGP & \multicolumn{1}{l}{$\begin{array}{l}\text { NEIC } \\
\text { USGS }\end{array}$} \\
\hline Fecha & & 21 Feb 1996 \\
Hora GMT & $12: 50: 56$ & $12: 51: 01$ \\
Latitud & $-09.68^{\circ}$ & $-09.593^{\circ}$ \\
Longitud & $-80.08^{\circ}$ & $-79.587^{\circ}$ \\
Profundidad & $8.1 \mathrm{~km}$ & $10 \mathrm{~km}$ \\
Magnitud & $7.2 \mathrm{Mw}$ & $7.5 \mathrm{Mw}$ \\
Azimut & - & $340^{\circ}$ \\
Buzamiento & - & $15^{\circ}$ \\
Deslizamiento & - & $97^{\circ}$ \\
\hline
\end{tabular}

Tabla 1: Parámetros hipocentrales del sismo de Chimbote 1996 recalculados por el IGP y reportados por el NEICUSGS. El mecanismo focal corresponde al reporte del Global CMT.

Los parámetros hipocentrales del terremoto de Chimbote de 1996 han sido tomados del reporte final recalculado del Instituto Geofísico del Perú [9]. El epicentro estuvo ubicado a $180 \mathrm{~km}$ al sur oeste de la ciudad de Chimbote, Ancash, Perú, donde fue percibido con intensidad de grado IV en la Escala Modificada de Mercalli. La magnitud reportada por el IGP fue de 7.2 
Mw, mientras que la magnitud reportada por el NEICUSGS fue de $7.5 \mathrm{Mw}$, en este estudio se calculó una magnitud de $7.6 \mathrm{Mw}$; otros parámetros de interés se aprecian en la Tabla 1.

\section{Características de la sismicidad}

El borde occidental de América del Sur se caracteriza por ser una de las regiones sísmicamente más activas en el mundo. El Perú forma parte de esta región y su actividad sísmica más importante está asociada al proceso de subducción de la placa de Nazca bajo la placa Sudamericana, generando frecuentemente sismos de magnitud elevada.

El mapa de la actividad sísmica ocurrida en la zona centro-norte del Perú durante el periodo 1970-2014 con eventos cuyo $\mathrm{M}>5.0 \mathrm{Mw}$ según el catálogo del United States of Geological Survey, USGS [10], se muestra en la Figura 1. La sismicidad con hipocentros de profundidad superficial, $\mathrm{h}<60 \mathrm{~km}$, se localiza principalmente entre la fosa marina y la línea de costa, con ocurrencia de sismos de magnitud elevada como, por ejemplo, en el norte de Lima en el año 1940, 8.0 Ms; en Ancash en el año 1966, 8.0 Ms y en Ancash en el año de 1970, 7.9 Mw.

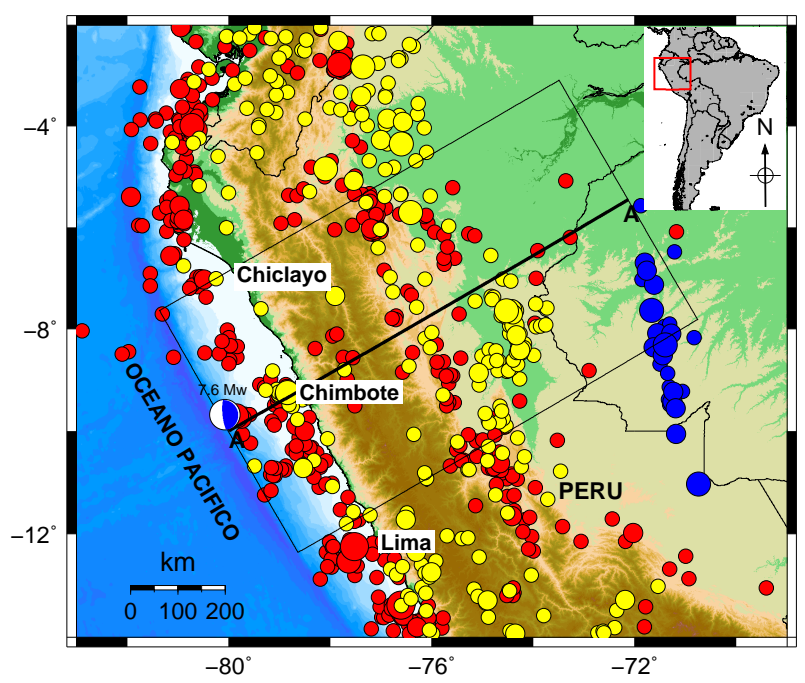

Figura 1: Mapa de sismicidad considerando $M>5.0 \mathrm{Mw}$ para la zona norte de Perú según el catálogo del NEIC 19702014. Color rojo: sismos superficiales $(h<60 \mathrm{~km})$, color amarillo: sismos intermedios ( $60 \mathrm{~km}<\mathrm{h}<300 \mathrm{~km}$ ) y color azul: sismos profundos $(\mathrm{h}>300 \mathrm{~km})$. El diagrama focal representa a la fuente sísmica del evento de Chimbote 1996.

Un perfil vertical de sismicidad con dirección perpendicular a la fosa marina, con un ancho de banda de la proyección de $600 \mathrm{~km}$ por una longitud de $1000 \mathrm{~km}$, de acuerdo al rectángulo indicado en la Figura 1 , se muestra en la Figura 2. Siguiendo el segmento $\overline{A A^{\prime}}$, la máxima profundidad de los sismos aumenta de Oeste a Este, con focos a menos de $50 \mathrm{~km}$ en la zona oceánica hasta $700 \mathrm{~km}$ de profundidad debajo del continente.

Entre 200 y $400 \mathrm{~km}$ de distancia horizontal desde el punto $\mathrm{A}$, origen del sistema de coordenadas de la Figura 2 , se observa una menor ocurrencia de sismos. A partir de la distancia de $400 \mathrm{~km}$, aumenta el número y la profundidad de los sismos, hasta una distancia horizontal de poco más de $700 \mathrm{~km}$ desde la fosa. Los sismos a estas profundidades indican el límite de la subducción de la placa de Nazca debajo la Sudamericana.

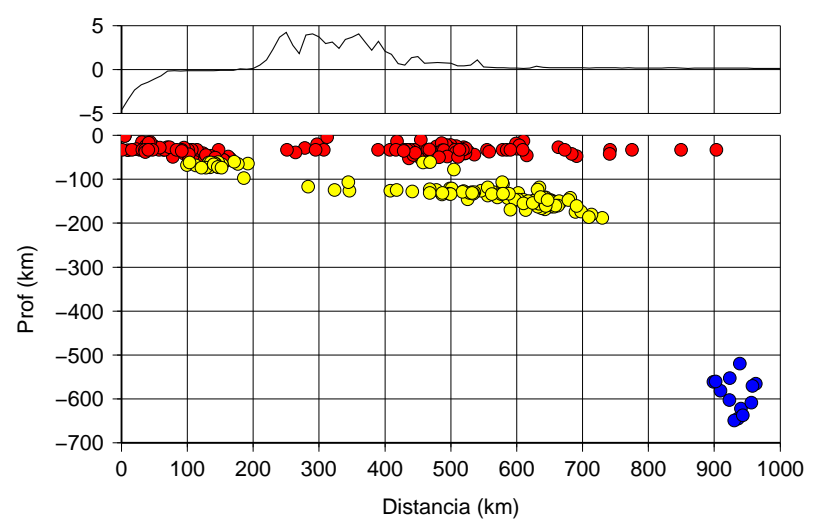

Figura 2: Perfil de sismicidad para el corte $\overline{A A^{\prime}}$ del mapa de sismicidad de la Figura 1. En color rojo se muestran los sismos superficiales, $\mathrm{h}<60 \mathrm{~km}$; en color amarillo, los sismos intermedios con profundidades que abarcan $60 \mathrm{~km}<\mathrm{h}$ $<300 \mathrm{~km}$ y en color azul, se muestran los sismos profundos, h $>300 \mathrm{~km}$. En la parte superior se muestra la proyección del perfil de la topografía.

De $100 \mathrm{~km}$ a $150 \mathrm{~km}$ de profundidad, los hipocentros se distribuyen alrededor de una línea con pendiente de $15^{\circ}$ a $20^{\circ}$ y a partir de esta profundidad el tope de la placa tectónica es casi horizontal, Figura 2. De los 200 $\mathrm{km}$ hasta los $500 \mathrm{~km}$ de profundidad no hay actividad sísmica. Los sismos de foco profundo se distribuyen entre los 500 y $700 \mathrm{~km}$. A profundidades mayores de los $700 \mathrm{~km}$ de profundidad cesa toda actividad sísmica.

El perfil de sismicidad de la Figura 2 muestra un proceso de subducción subhorizontal debido a la geometría casi horizontal de la zona de Wadati-Benioff entre los 200 y $700 \mathrm{~km}$ de distancia desde la fosa, punto $A$. 


\section{Adquisición de datos y procesamiento de las señales}

Para el cálculo del mecanismo focal del terremoto de Chimbote 1996, se ha utilizado datos de 15 estaciones sísmicas de banda ancha de la red IRIS [11] y GEOSCO$P E$ [12] para una distancia epicentral entre $30^{\circ}$ y $90^{\circ} \mathrm{con}$ una cobertura azimutal para el hemisferio norte y muy escasa en el hemisferio sur, ver Figura 3. Para distancias epicentrales menores a $30^{\circ}$ las ondas sufren fuertes perturbaciones por la estructura del manto superior y para distancias epicentrales mayores a $90^{\circ}$ las ondas sufren perturbaciones debido a que atraviesan el núcleo terrestre.

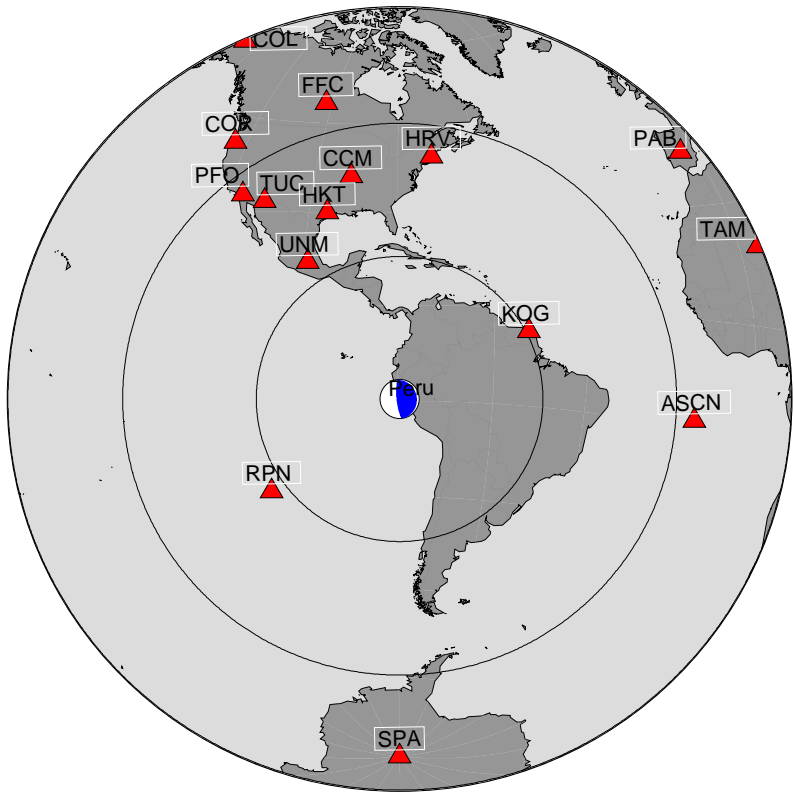

Figura 3: Distribución de las 15 estaciones telesísmicas usadas en la inversión. Cada círculo concéntrico representa una distancia epicentral de $30^{\circ}$. El diagrama focal representa la fuente sísmica del sismo de Chimbote 1996. Se observa que en el hemisferio norte hay una mayor de densidad de estaciones sísmicas.

Un sismograma está definido como una serie de tiempo $s(t)$ registrada por un sismógrafo en una ubicación particular a una distancia epicentral $\Delta$ respecto a la fuente, que corresponde al movimiento del suelo debido al arribo de las ondas sísmicas y puede ser expresado como la convolución de

$$
s(t)=u(t) * f(Q, t) * I(t),
$$

donde $u(t)$ es el movimiento de desplazamiento en la fuente, los efectos de superficie libre $f(Q, t)$ corresponden a la atenuación anelástica a lo largo de la trayectoria del rayo sísmico e $I(t)$ es la respuesta del instrumento 14 .

Las señales sísmicas de las estaciones de la Figura 3 han sido procesadas digitalmente, para lo cual se han removido las respuestas del instrumento mediante un proceso de deconvolución y se han filtrado las señales entre $2 \mathrm{mHz}$ y $100 \mathrm{mHz}$, o para el rango de periodos entre $10 \mathrm{~s}$ y $500 \mathrm{~s}$, para una ventana de tiempo de $80 \mathrm{~s}$ con un intervalo de muestreo de $0.5 \mathrm{~s}$. Finalmente, se ha realizado una rotación de los ejes horizontales para obtener las componentes radial y transversal, y así tomar en cuenta la fase SH. Para determinar el tiempo de arribo de la fase $\mathrm{P}, \mathrm{SH}$ y $\mathrm{pP}$ se han utilizado las tablas de Jeffreys-Bullen, luego se han corregido manualmente el tiempo de arribo de la fase $P$, para tener en cuenta las variaciones debido a las heterogeneidades de la corteza.

\section{Cálculo de las funciones de Green}

Una función de Green es la respuesta de un sistema cuando la señal de entrada es una perturbación unitaria. En este caso, la función de Green está representada por un sismograma sintético elemental, donde la perturbación unitaria está representada por los tensores elementales de momento sísmico que representan al sistema de fuerzas en la región de la fuente.

Se calcula las funciones de Green, en el dominio de la frecuencia usando la transformada de Fourier, para los 6 tensores de momento sísmico elementales localizados en un sistema de grillas sobre el plano de ruptura en torno al epicentro y a varias profundidades. Se escoge la profundidad que produce el error mínimo durante el proceso de inversión. Se ha considerado 512 puntos de la señal en el dominio del tiempo, potencia de 2, con una razón de muestreo de $0.5 \mathrm{~s}$. Se ha tomado en cuenta las ondas $\mathrm{P}$ (directas), pP (reflejadas) y SH (rotadas). Se ha asumido un modelo de 6 capas para la estructura de la corteza y el modelo de las velocidades de las ondas sísmicas en el norte del Perú [15], se ha añadido una capa de $4.2 \mathrm{~km}$ de espesor para modelar el comportamiento de las ondas sísmicas en el océano, ver Tabla 2. Se ha utilizado filtros $Q$ con una constante de tiempo de atenuación de $1 \mathrm{~s}$ para las ondas $\mathrm{P}$ y $4 \mathrm{~s}$ para las ondas $S$ en convolución con una función temporal de fuente compuesta por varios triángulos isósceles desplazados en el tiempo cuyas alturas se calculan mediante el proceso de inversión. 


\begin{tabular}{ccccr}
\hline$N$ & $v_{p}(\mathrm{~km} / \mathrm{s})$ & $v_{s}(\mathrm{~km} / \mathrm{s})$ & $\rho\left(\mathrm{g} / \mathrm{cm}^{3}\right)$ & $t(\mathrm{~km})$ \\
\hline \multicolumn{5}{c}{ Fuente } \\
\hline 1 & 1.50 & 0.00 & 1.02 & 4.2 \\
2 & 5.66 & 3.23 & 2.60 & 2.0 \\
3 & 5.92 & 3.38 & 2.60 & 8.0 \\
4 & 6.20 & 3.54 & 2.90 & 12.0 \\
5 & 6.44 & 3.68 & 3.38 & 8.0 \\
6 & 6.87 & 3.92 & 3.38 & 20.0 \\
7 & 7.92 & 4.52 & 3.37 & 0.0 \\
\hline \multicolumn{5}{c}{ Estación } \\
\hline 1 & 5.57 & 3.36 & 2.65 & 15.0 \\
2 & 6.50 & 3.74 & 2.87 & 18.0 \\
3 & 7.80 & 4.40 & 3.30 & 0.0 \\
\hline
\end{tabular}

Tabla 2: Modelo de la estructura de velocidades de las ondas sísmicas en la vecindad de la fuente, norte del Perú. Se ha agregado una capa adicional de $4.2 \mathrm{~km}$ de espesor para modelar el comportamiento de las ondas sísmicas en el océano. Para las estaciones telesísmicas se ha utilizado un modelo global.

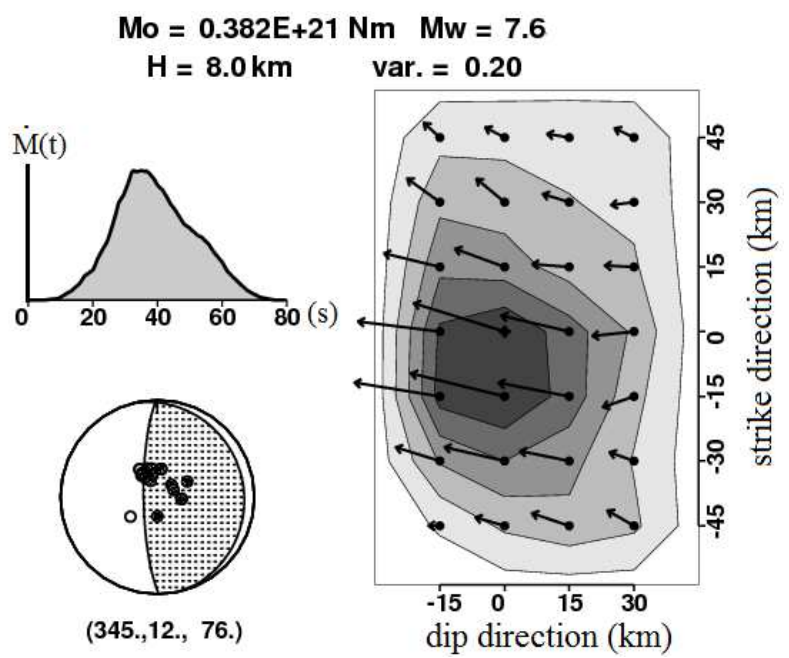

Figura 4: La función temporal de la fuente indica una duración del proceso de ruptura de $75 \mathrm{~s}$. El mecanismo focal está dado por strike $=345^{\circ}, d i p=12^{\circ}$ y rake $=76^{\circ}$. A la derecha se muestra la distribución de los vectores de desplazamiento. El error o varianza normalizada es de 0.20 .

\section{Inversión de las ondas telesísmicas}

Se ha utilizado el modelo numérico de Kikuchi y Kanamori [16] para modelar las formas de las ondas de volumen. Como condición inicial para la modelización de las formas de onda de volumen, se ha tomado los valores del mecanismo focal proporcionados por el The Global Centroid-Moment-Tensor Project, CMT [13]: azimut (strike) $=340^{\circ}$, buzamiento (dip) $=15^{\circ}$, ángulo de dislocación (rake) $=97^{\circ}$. Luego, el ángulo azimutal y el ángulo de buzamiento se han variado manualmente de grado en grado hasta encontrar el menor error o varianza. El ángulo de dislocación es variado por el modelo numérico en forma automática. La solución obtenida es similar a la propuesta por Ihmle y colaboradores [5].
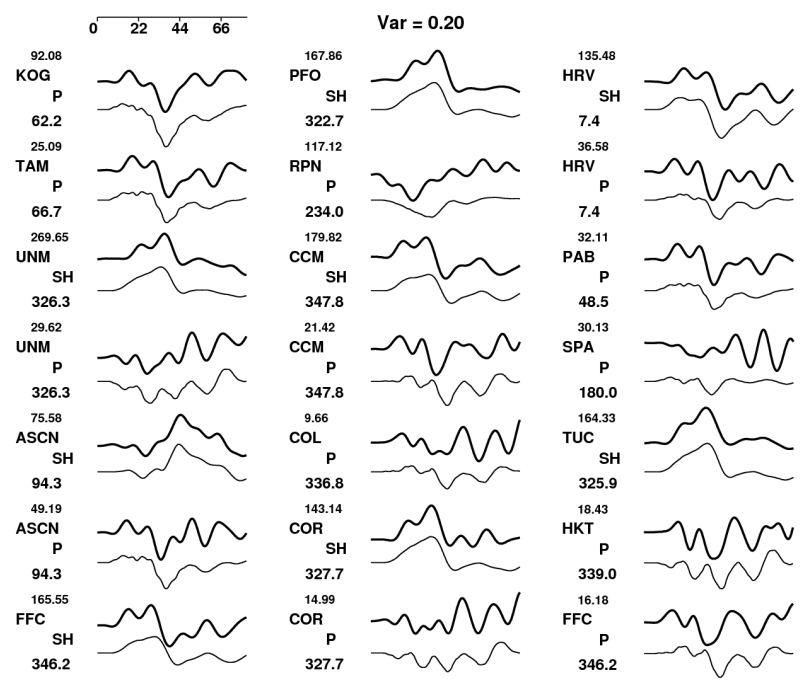

Figura 5: Comparación entre las señales observadas (superior) y sintéticas (inferior) para una ventana de tiempo de $80 \mathrm{~s}$. En general, hay una buena correlación. La leyenda a la izquierda de cada par de señales indica: amplitud (um), codigo de la estacion, tipo de señal $(\mathrm{P} \circ \mathrm{SH})$ y ángulo azimutal de la estacion con respecto al epicentro.

Luego de realizar la inversión de las formas de onda tal como se muestra en la Figura 4, se obtuvieron los siguientes parámetros del mecanismo focal: strike $=345^{\circ}$, $d i p=12^{\circ}$ y rake $=76^{\circ}$ para una profundidad de $8 \mathrm{~km}$, con un error o varianza normalizada de 0.20 que se muestra para diferentes profundidades en la Figura 6. El mecanismo focal obtenido es de tipo inverso. La función temporal de la fuente sísmica es suave, aunque, indica un proceso de ruptura complejo sin eventos secundarios prominentes. De acuerdo a Kikuchi y Kanamori [17] esta es una de las principales características de un tsunami earthquake. La duración total del proceso de ruptura es alrededor de $75 \mathrm{~s}$, un valor relativamente grande para un sismo de esta magnitud, lo que es característico de los tsunami earthquakes.

El momento sísmico total calculado es de $3.8 \times 10^{20}$ $\mathrm{Nm}$, correspondiente a una magnitud de momento de 7.6 $\mathrm{Mw}$, valor similar al obtenido por el USGS-NEIC 
(7.5 Mw), el IGP reportó una magnitud de 7.2 Mw. La longitud de ruptura fue alrededor de $90 \mathrm{~km}$ en la dirección del ángulo azimutal del plano de ruptura (Figura 4). Se observa una gran aspereza concentrada en un área de aproximadamente $30 \times 30 \mathrm{~km}^{2}$ en la zona central del área de ruptura y en el borde cercano a la fosa, la misma que corresponde al área de desplazamiento máximo o de mayor liberación de energía.

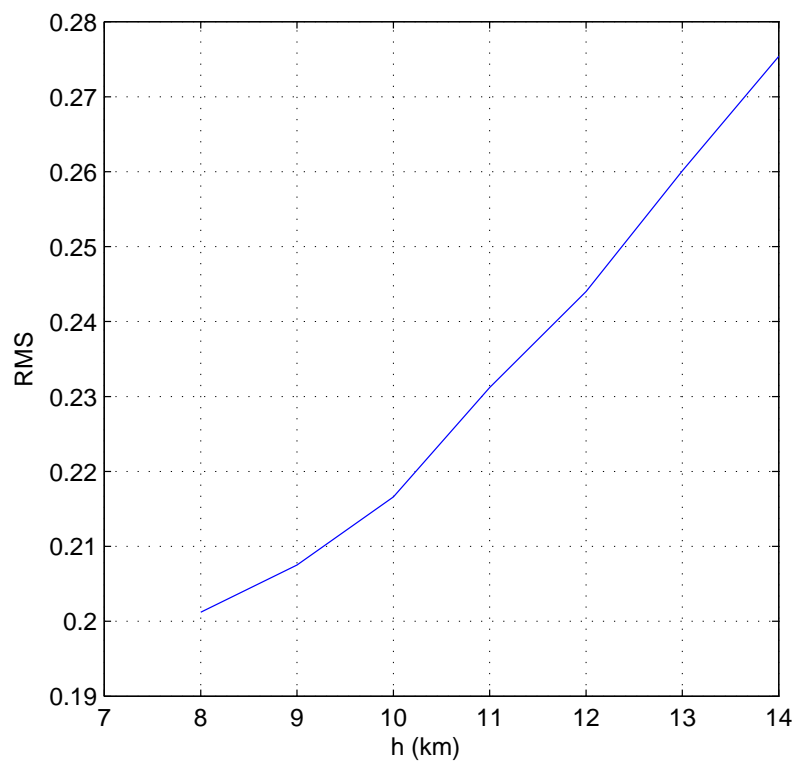

Figura 6: La curva RMS vs profundidad hipocentral $h$ indica que a una profundidad de $8 \mathrm{~km}$ se obtiene el menor error Para profundidades menores el modelo no trabaja debido a que parte del plano de ruptura estaría numéricamente por encima de la superficie oceánica.

En la Figura 5 se muestra una comparación entre las señales observadas y las señales sintéticas para una ventana de tiempo de $80 \mathrm{~s}$. En total hay 21 señales, 13 correspondientes a la componente $\mathrm{P}$ y 8 a la componente $\mathrm{SH}$; en general, se observa una buena correlación con un error o varianza normalizada de 0.20 .

Teóricamente, la velocidad de ruptura es el $75 \%$ de la velocidad de propagación de la onda S. Si la longitud de la ruptura fue de $90 \mathrm{~km}$ y la duración total del proceso de ruptura fue $75 \mathrm{~s}$, entonces la velocidad de ruptura promedio fue de $1.2 \mathrm{~km} / \mathrm{s}$, lo que corresponde a un proceso de ruptura lento, considerando que la velocidad de ruptura promedio de sismos superficiales es de $3.0 \mathrm{~km} / \mathrm{s}$, característico de los tsunami earthquake. Adicionalmente, variando la velocidad de ruptura en el modelo de inversión, la velocidad de ruptura que genera el menor error es $1.4 \mathrm{~km} / \mathrm{s}$, tal como se muestra en la Figura 7.

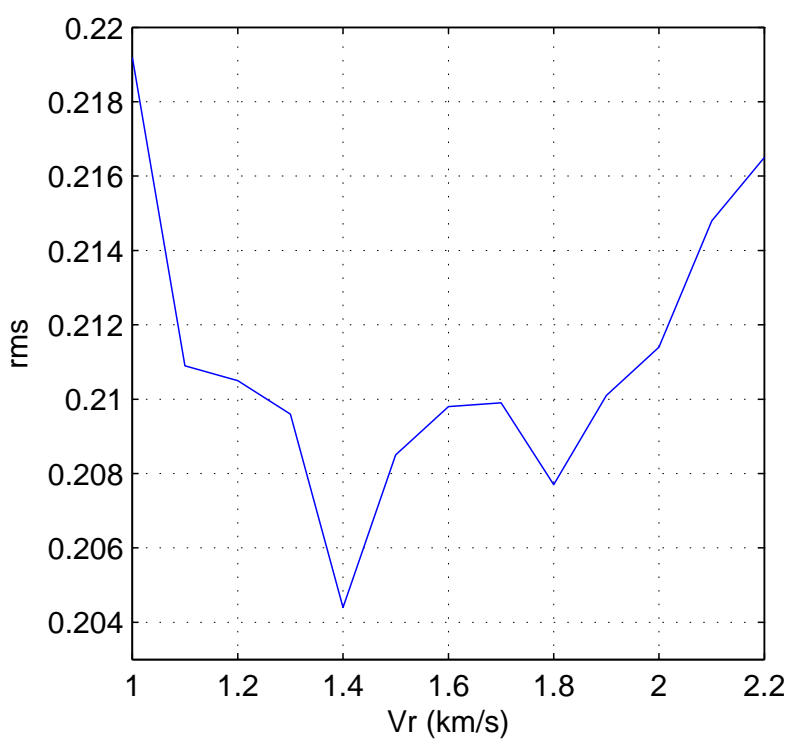

Figura 7: La curva RMS vs velocidad de propagación $v r$ indica que a una velocidad de $1.4 \mathrm{~km} / \mathrm{s}$ se obtiene el menor error o residual.

\section{Deformación y desplazamiento cosísmico}

Para obtener el modelo de deformación, se utilizó la teoría de Okada [8] para un medio elástico, homogéneo, isotrópico y semi-infinito. Se consideraron 28 subfuentes sísmicas de dimensiones de $15 \times 15 \mathrm{~km}^{2}$, distribuídos sobre el plano de ruptura, Figura 8 , con una dislocación, slip y ángulo de desplazamiento, rake, variables, ver la Tabla 3. Los parámetros del mecanismo focal tales como la azimut o strike y el ángulo de buzamiento o dip, son tomados del resultado de la inversón y son iguales para cada sub-fuente. La magnitud de la dislocacion es inversamente proporcional al coeficiente de rigidez del medio elástico, de tal forma que para un coeficiente de rigidez igual a $2.0 \times 10^{10} \mathrm{~N} / \mathrm{m}^{2}$, valor propuesto por Ihmle y colaboradores [5] resulta en una dislocacion máxima de $6.62 \mathrm{~m}$.

Se asume que la generación cosísmica de la deformación sobre el fondo oceánico produce una deformación inmediata e idéntica en la superficie oceánica debido a la incompresibilidad del fluido. Como el océano es un fluido, la deformación no conserva su forma y el agua empieza a fluir hacia los costados creando una onda de maremoto que se propaga en todas las direcciones. La Figura 9 muestra la componente vertical del campo de deformación cosísmica. De acuerdo al perfil de deformación según el corte $A A^{\prime}$, el máximo levantamiento en la trayectoria del perfil fue de $2.21 \mathrm{~m}$ y la máxima 
subsidencia o hundimiento fue de $0.54 \mathrm{~m}$.

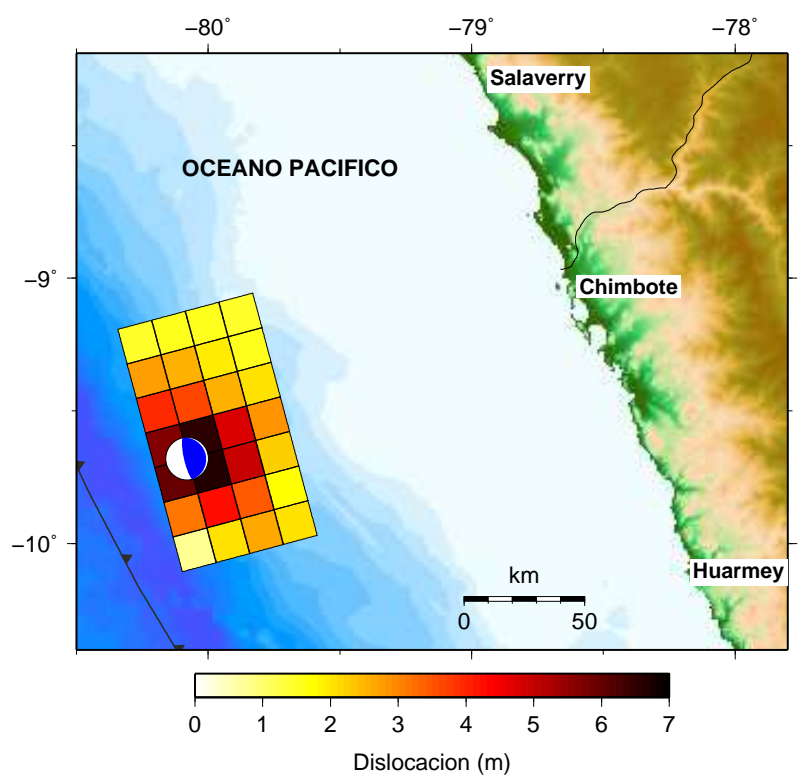

Figura 8: Distribución de las sub-fuentes. Cada fuente unitaria tiene dimensiones de $15 \times 15 \mathrm{~km}^{2}$. El mecanismo focal está representado por el diagrama focal.

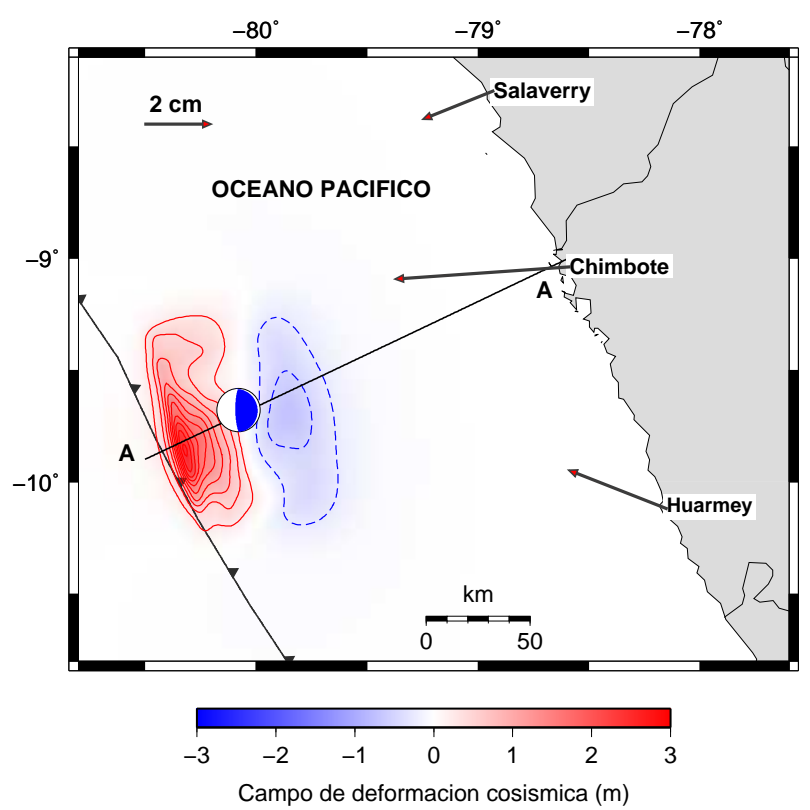

Figura 9: Componente vertical del campo de deformación cosísmica. El color rojo indica el levantamiento y el color azul indica la subsidencia. El intervalo de las curvas de nivel representan $0.25 \mathrm{~m}$. Las flechas representan a los vectores de desplazamiento cosísmico horizontal.

Debido a la lejanía de la fuente sísmica que se encuentra más cerca a la fosa marina con respecto a la costa, alrededor de $180 \mathrm{~km}$, el desplazamiento de la ciudad de Chimbote no es apreciable, la magnitud de la componente horizontal de desplazamiento simulado fue de $5.3 \mathrm{~cm}$, ver la Figura 9 .

\begin{tabular}{lrcrcr}
\hline$N$ & Lat $\left({ }^{\circ}\right)$ & Lon $\left({ }^{\circ}\right)$ & $h(\mathrm{~km})$ & $u(\mathrm{~m})$ & rake $\left({ }^{\circ}\right)$ \\
\hline 1 & -10.0035 & -79.7156 & 14.24 & 2.09 & 59.98 \\
2 & -10.0377 & -79.8431 & 11.12 & 2.69 & 71.38 \\
3 & -10.0718 & -79.9706 & 8.00 & 2.08 & 73.76 \\
4 & -10.1060 & -80.0980 & 4.88 & 0.78 & 89.52 \\
5 & -9.8732 & -79.7506 & 14.24 & 1.77 & 70.28 \\
6 & -9.9074 & -79.8780 & 11.12 & 3.44 & 78.48 \\
7 & -9.9415 & -80.0055 & 8.00 & 4.23 & 76.99 \\
8 & -9.9757 & -80.1329 & 4.88 & 3.16 & 72.66 \\
9 & -9.7429 & -79.7855 & 14.24 & 2.25 & 109.18 \\
10 & -9.7771 & -79.9129 & 11.12 & 4.93 & 79.10 \\
11 & -9.8112 & -80.0404 & 8.00 & 6.62 & 76.19 \\
12 & -9.8454 & -80.1678 & 4.88 & 5.93 & 80.77 \\
13 & -9.6126 & -79.8204 & 14.24 & 2.86 & 96.05 \\
14 & -9.6467 & -79.9478 & 11.12 & 4.78 & 76.83 \\
15 & -9.6809 & -80.0753 & 8.00 & 6.52 & 72.70 \\
16 & -9.7151 & -80.2028 & 4.88 & 5.64 & 82.99 \\
17 & -9.4823 & -79.8553 & 14.24 & 2.00 & 87.17 \\
18 & -9.5164 & -79.9828 & 11.12 & 2.53 & 85.61 \\
19 & -9.5506 & -80.1102 & 8.00 & 3.61 & 69.92 \\
20 & -9.5847 & -80.2377 & 4.88 & 3.95 & 76.29 \\
21 & -9.3520 & -79.8902 & 14.24 & 1.59 & 97.34 \\
22 & -9.3861 & -80.0177 & 11.12 & 1.90 & 72.79 \\
23 & -9.4203 & -80.1451 & 8.00 & 2.50 & 50.35 \\
24 & -9.4544 & -80.2726 & 4.88 & 2.75 & 54.20 \\
25 & -9.2217 & -79.9251 & 14.24 & 1.48 & 64.57 \\
26 & -9.2558 & -80.0526 & 11.12 & 1.54 & 80.58 \\
27 & -9.2900 & -80.1800 & 8.00 & 1.51 & 62.28 \\
28 & -9.3241 & -80.3075 & 4.88 & 1.49 & 47.35 \\
\hline
\end{tabular}

Tabla 3: Distribución de los vectores de dislocación, donde $u$ representa la magnitud de la dislocación (slip) y rake representa el ángulo de dislocación, para cada sub-fuente.

\section{Modelado numérico del maremoto}

Se utilizó una versión modificada del modelo numérico TUNAMI-N2 [18], para 4 grillas computacionales anidadas, en la grilla de propagación se utilizó un modelo lineal para una grilla batimétrica regular en coordenadas esféricas basado en la teoría hidrodinámica de aguas someras y en la grilla de inundación se utilizó un modelo no-lineal para una batimetría más densa, con una celda unitaria de $32 \mathrm{~m}$ de resolución, en coordenadas cartesianas. Las ecuaciones diferenciales de continuidad y conservación de momentum lineal, ecuaciones 
2 a 4, son integradas numéricamente usando el método de diferencias finitas bajo el esquema numérico del salto de rana. Se establece una condición de estabilidad numérica CFL menor a 0.8 para evitar inestabilidades numéricas.

$$
\begin{gathered}
\frac{\partial \eta}{\partial t}+\frac{\partial M}{\partial x}+\frac{\partial N}{\partial y}=0 \\
\frac{\partial M}{\partial t}+\frac{\partial}{\partial x}\left(\frac{M^{2}}{D}\right)+\frac{\partial}{\partial x}\left(\frac{M N}{D}\right)=-g D \frac{\partial \eta}{\partial x} \\
-\frac{g n^{2}}{D^{7 / 3}} M \sqrt{M^{2}+N^{2}} \\
\frac{\partial N}{\partial t}+\frac{\partial}{\partial x}\left(\frac{M N}{D}\right)+\frac{\partial}{\partial x}\left(\frac{N^{2}}{D}\right)=-g D \frac{\partial \eta}{\partial x} \\
-\frac{g n^{2}}{D^{7 / 3}} N \sqrt{M^{2}+N^{2}}
\end{gathered}
$$

donde

$$
\begin{aligned}
M & =\int_{-h}^{\eta} u d z \\
N & =\int_{-h}^{\eta} v d z \\
D & =\eta+h
\end{aligned}
$$

$M$ y $N$ representan la descarga de flujo para la dirección $x$ e $y$ respectivamente, $\eta$ es el nivel de agua y $h$ es la profundidad del agua con respecto al nivel medio del mar.

Las condiciones iniciales se basan en la teoría de deformación de Okadacite8. Las condiciones de frontera en la costa para el modelo lineal para la grilla de propagación, suponen una pared vertical, mientras que para el modelo no-lineal para la grilla de inundación, se utilizó una condición de frontera móvil.

El modelo numérico no toma en cuenta los efectos de dispersión que generen efectos no-lineales, que para este caso, por tratarse de un evento de campo cercano, no son apreciables.

Se han obtenido los mareogramas sintéticos en la bahía de Chimbote y en el puerto de Salaverry que se muestran en la Figura 10. Los resultados muestran una máxima altura de ola de $1.81 \mathrm{~m}$ y un tiempo de arribo de la primera onda negativa de $60 \mathrm{~min}$ en Chimbote. Los registros mareográficos de Chimbote y Salaverry son analógicos y de baja resolución, no pudiéndose realizar una comparación para efectos de validación. El mareograma observado en Chimbote se saturó en el minuto 69 después de ocurrido el sismo y dejó de registrar por el lapso de una hora, cuando la altura de la ola llegó a $1.3 \mathrm{~m}$ aproximadamente [7]). De acuerdo a Satake y Tanioka [19], el mareógrafo de Salaverry registró el maremoto con una máxima amplitud de $77 \mathrm{~cm}$ a los 90 min después de ocurrido el sismo. El mareograma sintético de Salaverry muestra una amplitud máxima de $1.04 \mathrm{~m}$ y un tiempo de arribo de la primera ola de 83 $\min$.
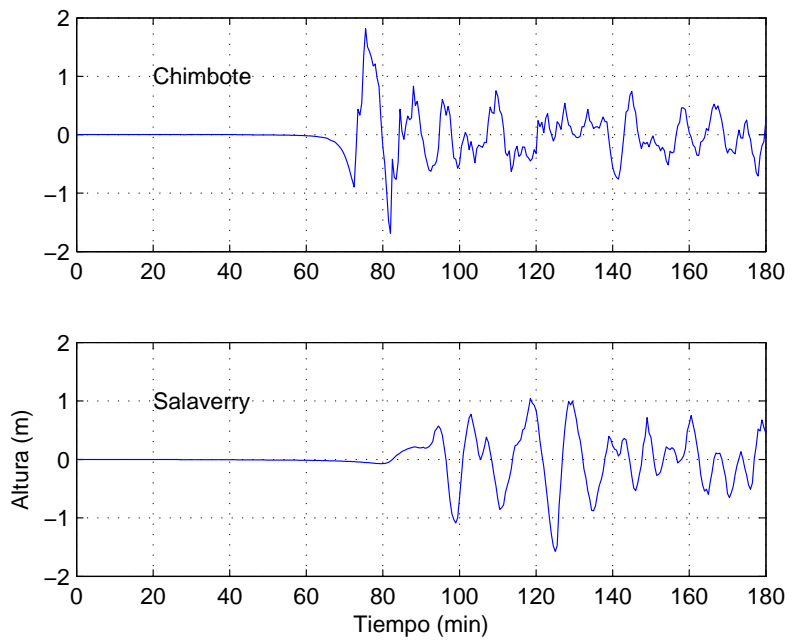

Figura 10: Mareogramas sintéticos para Chimbote y Salaverry. La máxima amplitud en Chimbote es $1.81 \mathrm{~m}$ con respecto al nivel medio del mar, se observa un retiro inicial del mar de poco menos de $1 \mathrm{~m}$. La máxima amplitud en Salaverry es $1.04 \mathrm{~m}$.

\section{Conclusiones}

El sismo de Chimbote 1996, está clasificado como un sismo tsunamigénico o tsunami earthquake debido a la pequeña velocidad de ruptura $(1.2 \mathrm{~km} / \mathrm{s}$ a $1.4 \mathrm{~km} / \mathrm{s})$, a la larga duración relativa del proceso de ruptura (75 s) y a los efectos del impacto del maremoto, los cuales son mayores a lo que se espera de un sismo de magnitud 7.6 Mw.

La función temporal de la fuente sísmica del terremoto de Chimbote de 1996, es liza o suave e indica un proceso de ruptura complejo sin eventos secundarios prominentes. La longitud promedio de la ruptura es de alrededor de $90 \mathrm{~km}$; sin embargo, la aspereza principal está contenida en un área aproximada de $30 \times 30 \mathrm{~km}^{2}$. 
La fuente sísmica estuvo ubicada muy cerca a la fosa marina y a una profundidad de $8 \mathrm{~km}$, en la región de acreción o de crecimiento por adición de material litosférico sedimentario; por lo que se ha elegido una constante de rigidez del medio elástico de $2 \times 10^{10} \mathrm{~N} / \mathrm{m}^{2}$.

El momento sísmico escalar promedio calculado por el método de inversión de formas de ondas telesísmicas es de $3.8 \times 10^{20} \mathrm{Nm}$ y corresponde a una magnitud de 7.6 Mw. El máximo valor de la dislocación o slip es de $6.62 \mathrm{~m}$, un valor relativamente grande para un sismo de esta magnitud. Esto explica la generación del maremoto con máximas amplitudes de las olas de aproximadamen- te 2 a $3 \mathrm{~m}$.

La duración del proceso de ruptura fue de alrededor de $75 \mathrm{~s}$ y la velocidad de ruptura promedio de 1.2 $\mathrm{km} / \mathrm{s}$, esto implica la ocurrencia de un sismo con un proceso de ruptura lento, característico de los sismos tsunamigénicos.

El mareograma sintético de Chimbote indica un tiempo de arribo de la primera onda negativa de 60 minutos y una altura máxima de $1.8 \mathrm{~m}$. La señal mareográfica observada es analógica y presenta una saturación cuando la ola llegó a una altura de $1.3 \mathrm{~m}$ con un gap de datos por un lapso de una hora.

\section{Referencias}

[1] L. Ocola y P. Huaco; Informe Técnico, Instituto Geofísico del Perú, Lima (2006).

[2] H. Kanamori, Phys. Earth Planet. Interiors 6, 346 (1972).

[3] E. Okal y A. Newman, Phys. Earth Planet. Interiors 124, 45 (2001).

[4] H. Tavera, Mecanismo focal de terremotos y Sismotectónica, Tesis de Doctorado, Universidad Complutense de Madrid, Madrid (1998).

[5] P. Ihmle, J. Gomez, P. Heinrich y S. Guibourg, Geophys. Res. Lett. 25, 2691 (1998).

[6] P. Heinrich, F. Schindele, S. Guibourg y P. Ihmle, Geophys. Res. Lett. 25, 2691 (1998).

[7] J. Bourgeois, C. Petroff, H. Yeh, V. Titov, C. Synolakis, B. Benson, J. Kuroiwa, J. Lander y E. Norabuena, Pure \& Appl. Geophys. 154, 513 (1999).

[8] Y. Okada, Bull. Seismol. Soc. America 82, 1018 (1992).

[9] Instituto Geofísico del Perú, www.igp.gob.pe.

[10] US Geological Survey, earthquake.usgs.gov/earthquakes/map
[11] Incorpored Research Institutions for Seismology,

WWW.iris.edu.

[12] Global Netwok of Broad Band Seismic Stations, geoscope.ipgp.fr/index.php/en/.

[13] The Global Centroid-Moment-Tensor Project, WWW.globalcmt . org

[14] A. Udías, R. Madariaga y E. Buforn; Source Mechanism of Earthquakes: Theory and Practice, Cambridge University Press, Cambridge (2004).

[15] J. Villegas y H. Tavera, Bol. Soc. Geol. Perú 104, 81 (2010).

[16] M. Kikuchi y H. Kanamori, Notes on Teleseismic Body-Wave Inversion Program, Tokio, (2003) www.eri.u-tokyo.ac.jp/ETAL/KIKUCHI

[17] M. Kikuchi y H. Kanamori, Pure \& Appl. Geophys. 144(3-4), 441 (1995).

[18] S. Koshimura, TUNAMI Code, Numerical Analysis Model for Investigation of Tsunamis, Tohoku University, Sendai (2009).

[19] K. Satake y Y. Tanioka, Pure \& Appl. Geophys. 154, 467 (1999). 